- Notes -

\title{
Enrichment of Triglycerides with Polyunsaturated Fatty Acids Using Cuprous Ion Complex Formation Extraction
}

\author{
Ryousuke MAETA ${ }^{1}$ and Satoru KATO ${ }^{2}$ \\ ${ }^{1}$ : Department of Urban System Science, Tokyo Metropolitan University \\ 2: Department of Applied Chemistry, Tokyo Metropolitan University, \\ 1-1 Minamiohsawa, Hachiohji 192-0397, Japan
}

(Received December 28, 2009, Accepted February, 1, 2010)

\begin{abstract}
Polyunsaturated fatty acids (PUFA), such as eicosapentaenoic acid and docosahexaenoic acid, contained in triglycerides were concentrated from $46 \%$ to $69 \%$ by cuprous ion complex formation extraction, which uses an extraction solvent consisting of ethanol, cupric nitrate and metallic copper. The equilibration period was as long as six hours. More than $90 \%$ of the PUFA in the feed triglycerides was extracted. The residual copper in the enriched triglycerides was as low as $10 \mathrm{ppm}$ after four water washings.
\end{abstract}

\section{Introduction}

Eicosapentaenoic acid (EPA, 20:5) and docosahexaenoic acid (DHA, 22:6) are the most essential long-chain n-3 polyunsaturated fatty acids (PUFAs) present in fish oils [1]. They are recognized to have special pharmacological and physiological effects on human health [2-5]. Fish oil in its natural triacylglycerol form and fish oil ethyl esters have been widely marketed in drug and health food stores [6]. Of these, the triglycerides are more readily absorbed in human bodies than the esters [6]. Although the PUFA esters can be purified up to $95 \%$ using vacuum distillation, the purity of the PUFA triglycerides is limited to $45 \%$ due to the low yield of the lipase-catalyzed trans-esterification reaction [1]. Nakano et al. [7] demonstrated that PUFA ethyl esters can be extracted using metallic copper and a copper (II) salt dissolved in water. However, the extraction of the PUFA triglycerides using cuprous ion complex formation has not been reported in the literature. As for the solvent, copper (II) salts, such as cupric nitrate $\mathrm{Cu}\left(\mathrm{NO}_{3}\right)_{2}$ are highly soluble in water. On the other hand, the triglycerides are only very slightly soluble in water. Another solvent candidate could be ethanol wherein cupric nitrate is highly soluble, and it is suitable as an extraction solvent in food production processes. The purpose of the present investigation is to show that the PUFA triglycerides can be purified up to $69 \%$ using cuprous ion complex formation extraction with ethanol as the solvent.

\section{Experimental}

\subsection{Reagents}

The 46 \% PUFA triglyceride feed stock was supplied by Raffinee International Co., Ltd., and was used 
without further purification. Reagent grade ethanol, cupric nitrate $\mathrm{Cu}\left(\mathrm{NO}_{3}\right)_{2} \cdot 3 \mathrm{H}_{2} \mathrm{O}$, hexadecane, acetone and $0.2 \mathrm{~mm}^{\phi}$ copper powder were purchased from Kanto Chemical Co., Ltd. Water was purified by distillation and ion exchange. A methanol solution containing $0.2 \mathrm{M}$ phenyltrimethylammonium hydroxide (PTAH) was supplied by Shiwa Kougyo Co., Ltd. The PTAH methanol solution catalyzes the following transesterification reaction, and produces fatty acid methyl esters, which were analized:

$$
\text { Triglycerides }+3 \mathrm{CH}_{3} \mathrm{OH} \leftrightharpoons \text { methyl esters }+ \text { glycerol }
$$

\subsection{Methods}

The $\mathrm{Cu}\left(\mathrm{NO}_{3}\right)_{2}$ ethanol solution was stored for one day, and the transparent upper solution was used for the extraction experiments. Before equilibration, the ethanol solution was degassed at $10 \mathrm{kPa}$ for $10 \mathrm{~min}$. using a suction pump, because the degassing operation is required to produce stable extraction data [8]. Using a centrifugal precipitation glass tube, the triglyceride oil and the extraction solvent consisting of ethanol, $\mathrm{Cu}\left(\mathrm{NO}_{3}\right)_{2} \cdot 3 \mathrm{H}_{2} \mathrm{O}$ and copper powder were shaken in a constant temperature water bath at a speed of $2 \mathrm{~s}^{-1}$. After extraction, the mixture was separated using a centrifuge at $3000 \mathrm{rpm}$ for $20 \mathrm{~min}$. The settled upper oil phase was washed with water and again centrifuged. A $0.2 \mathrm{ml}$ triglyceride aliquot of the final upper oil phase was taken and transferred to a glass bottle. The triglyceride oil was diluted with acetone and hexadecane. To the sample mixture, $0.3 \mathrm{ml}$ of a $0.2 \mathrm{M}$ PTAH methanol solution was added, and the triglyceride was transformed into fatty acid methyl esters with stirring for $30 \mathrm{~min}$. The mixture after the transesterification was analyzed by gas chromatograph using a Shimazu GC-14A equipped with a fire ionized detector. A 25 m Ulbon HR-SS-10 column from Shinwa Chemical Ind., Ltd., was used for the separation of the methyl esters.

Meanwhile, during the recovery operations for the PUFA triglycerides, the equilibrated and settled lower ethanol phase was separated using a centrifuge and heated. The settled upper triglyceride phase was washed four times with water, and the drained water phases were analyzed using atomic absorption spectrophotometer to determine the amount of residual copper. The final triglyceride oil was burned and diluted with water to determine the copper residue. Table 1 lists the experimental conditions.

Table 1 Experimental conditions in extraction.

\begin{tabular}{ll}
\hline Temperature & $288 \mathrm{~K}$ \\
Triglyceride & $3 \mathrm{~g}$ \\
Ethanol phase & $4 \mathrm{~cm}^{3}$ \\
$\quad \mathrm{Cu}\left(\mathrm{NO}_{3}\right)_{2}$ & $0.5-2 \mathrm{~mol} / \mathrm{dm}^{3}$ \\
Metallic copper & $2.5 \mathrm{~mol} / \mathrm{dm}^{3}$ \\
Stirring speed & $2 \mathrm{~s}^{-1}$ \\
Extraction time & $1-6 \mathrm{~h}$ \\
\hline
\end{tabular}

\section{Results and Discussion}

\subsection{Extraction rates}

In Fig. 1, the concentrations of EPA and DHA contained in the triglycerides (EPA-TG and DHA-TG, respectively) in the ethanol phase, $C_{\mathrm{i}, \mathrm{S}}$, are plotted versus the extraction time, t, where $C_{\mathrm{i}, \mathrm{S}}$ was calculated from the decreased amount of EPA and DHA in the oil phase after extraction. The EPA and DHA were transferred from the oil phase to the ethanol phase, because the triglycerides containing them were extracted. Figure 1 shows that extraction equilibrium is achieved within six hours for EPA-TG and DHA-TG. Figure 1 also includes the concentrations of 1,5-hexadiene and the EPA ethyl ester (EPA-Et) in 
the aqueous phase [9], as measured by the same method. Figure 1 demonstrates that high molecular-weight substances, such as the triglycerides and EPA-Et, require a 50 times longer equilibration period than that for 1,5-hexadiene. The slow extraction rate may arise from the slow $\mathrm{Cu}(\mathrm{I})$-complex formation [8].

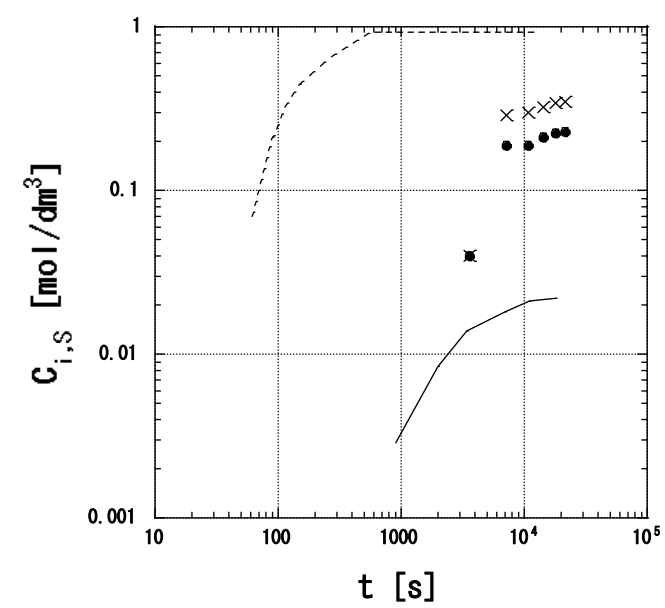

Fig. 1 Relationships between the solute concentration in the solvent phase, $C_{i, s}$, and the extraction time, t; $(\times)$ EPA-TG and (O) DHA-TG in the ethanol phase, $(-)$ EPA-Et and $(--)$ 1,5-hexadiene in the

\subsection{Extraction equilibria} aqueous phase [7].

In Fig. 2, the distribution ratio, $D_{\mathrm{i}}$, is plotted versus the initial concentration of $\mathrm{Cu}\left(\mathrm{NO}_{3}\right)_{2}$ in the ethanol phase, $C_{\mathrm{Cu}(I I), \text { ini. }}$.

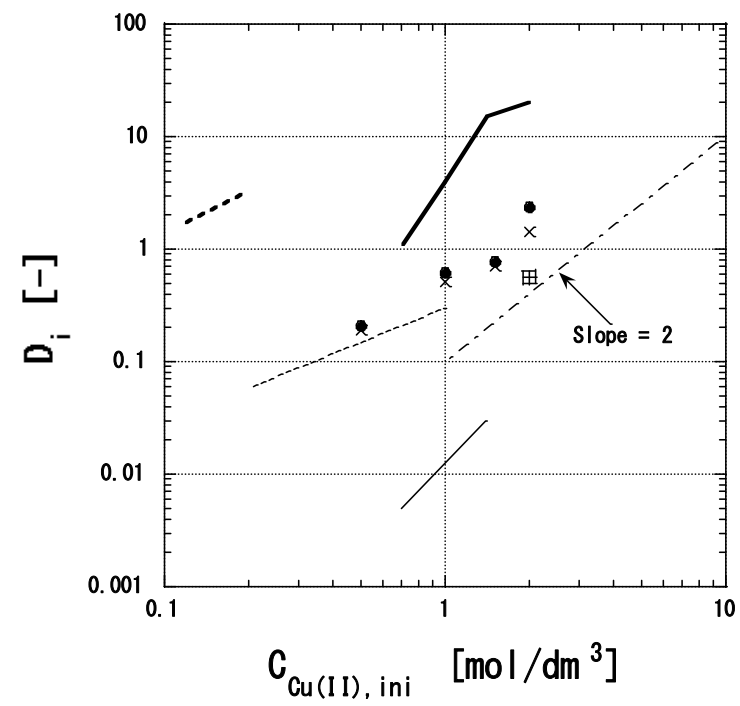

Fig. 2 Relationships between the distribution ratio, $D_{i}$, and the initial concentration of cupric nitrate; $(\times)$ EPA-TG and (@) DHA -TG in ethanol at $288 \mathrm{~K},(\square)$ EPA-TG and (+) DHA-TG in methanol at 288; (thick solid line) EPA-Et in water at $288 \mathrm{~K}$ [9]; (thin solid line) styrene in water at $288 \mathrm{~K}$ [8]; (thin dotted line) 1,5-hexadiene in water at $298 \mathrm{~K}$ [10]; and (thick dotted line) styrene in methanol at $298 \mathrm{~K}$ [8]. 
Di is defined as follows:

$$
D_{\mathrm{i}}=\frac{C_{\mathrm{i}, \mathrm{S}}}{C_{\mathrm{i}, \mathrm{oil}}}
$$

where $C_{i, o i l}$ denotes the concentration of the solute $\mathrm{i}$ in the oil phase. The extraction of the PUFA triglycerides, PUFA-TG, involves the following steps [7, 8]:

$$
\begin{aligned}
& (\text { PUFA - TG })_{\text {oil }} \leftrightharpoons(\text { PUFA }- \text { TG })_{\mathrm{S}} \\
& \mathrm{Cu}(0)+\mathrm{Cu}(\mathrm{II}) \leftrightharpoons 2 \mathrm{Cu}(\mathrm{I}) \\
& (\mathrm{PUFA}-\mathrm{TG})_{\mathrm{S}}+\mathrm{nCu}(\mathrm{I}) \leftrightharpoons \mathrm{PUFA}-\mathrm{TG}-\mathrm{Cu}(\mathrm{I})_{\mathrm{n}}
\end{aligned}
$$

The equilibrium constant for reaction (4) is as low as $10^{-6}$ [9]. Figure 2 shows that the distribution ratio of the triglycerides including EPA and DHA increases with increasing $C_{C u(I I), i n i}$. This arises from the PUFA-TG accumulation in the ethanol phase not only from the dissolution step shown in Eq. (3), but also from the complex formation step shown in Eq. (5). From slope analysis, the following formula can be obtained for the distribution ratio [8];

$$
D_{\mathrm{i}}=K C_{\mathrm{Cu}(\mathrm{II}), \mathrm{S}}{ }^{\mathrm{n} / 2}
$$

where $K$ denotes a constant. Assuming the relationship, $\mathrm{C}_{\mathrm{Cu}(\mathrm{II}), \mathrm{S}}=\mathrm{C}_{\mathrm{Cu}(\mathrm{II}), \mathrm{ini}}, n=2.0$ and 3.0 were, respectively, obtained for EPA-TG and DHA-TG using the data shown in Fig. 2; therefore, the unsaturated carbon-carbon double bonds of EPA-TG and DHA-TG were not saturated with $\mathrm{Cu}(\mathrm{I})$. The complex formation constant of DHA-TG is greater than that of EPA-TG, because $n_{D H A}>n_{E P A}$, where $n_{D H A}$ and $n_{E P A}$, respectively, denote the number of $\mathrm{Cu}(\mathrm{I})$ ions forming complexes with the DHA-TG and EPA-TG molecules. As shown in Fig. 2, $D_{i}$ for EPA-Et in water is greater than that for EPA-TG in ethanol. This is because EPA-Et is more soluble in water than EPA-TG in ethanol. The slope for EPA-Et in water is four, and is greater than for EPA-TG in ethanol, because the fatty acid carbon chains in the triglyceride molecules may hinder access of $\mathrm{Cu}(\mathrm{I})$ to the EPA chain. Furthermore, the $D_{\mathrm{i}}$ values for styrene in water [8] and 1,5-hexadiene in water [10] are plotted in Fig. 2. Figure 2 shows that the $D_{\mathrm{i}}$ values decrease with the decreasing number of carbon-carbon double bonds. In Fig. 3, the equilibrium extraction ratio, $E_{i}$, is plotted versus $C_{\mathrm{Cu}(\mathrm{II}) \text {,ini. }}$. The extraction ratios of PUFA-TG increase with increasing $C_{\mathrm{Cu}(\mathrm{II}) \text {,ini }}$ up to $90 \%$ at $C_{\mathrm{Cu}(\mathrm{II}), \text { ini }}$ $=2 \mathrm{~mol} / \mathrm{dm}^{3}$.

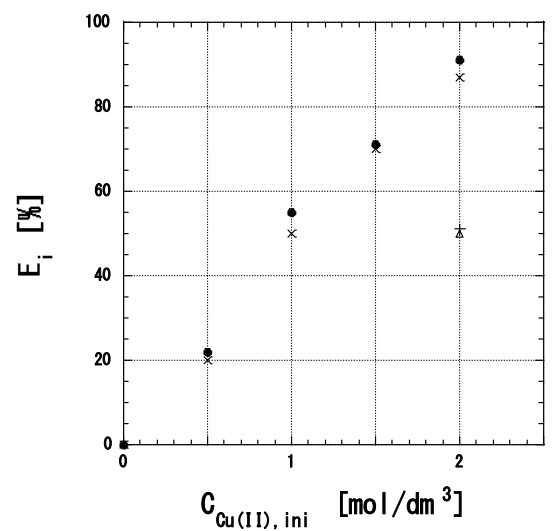

Fig. 3 Relationships between the equilibrium extraction ratio, $E_{i}$, and $C_{\mathrm{Cu}(I), \text { ini }}$; $(\times)$ EPA-TG and DHA-TG in ethanol; $(\triangle)$ EPA-TG and $(+)$ DHA-TG in methanol at $288 \mathrm{~K}$. 


\subsection{Effect of extraction solvents and temperature}

To examine the effect of the extraction solvents, Fig. 2 includes the $D_{i}$ values for EPA-TG and DHA-TG in methanol at $288 \mathrm{~K}$. Figure 2 shows that the distribution ratios in methanol are lower than those in ethanol. In Fig. 2, the $D_{i}$ values for styrene in methanol at $298 \mathrm{~K}$ are also plotted showing that the $D_{i}$ values of styrene in water are much lower than those in methanol. In fact, the PUFA triglycerides were not extracted by water even at $C_{\mathrm{Cu}(\mathrm{II}) \text {,ini }}=2 \mathrm{~mol} / \mathrm{dm}^{3}$ and $288 \mathrm{~K}$. Figure 3 includes the $E_{i}$ values of EPA-TG and DHA-TG in methanol at $288 \mathrm{~K}$. Figure 3 demonstrates that the extraction ratios for PUFA-TG in methanol are lower than those in ethanol. Table 2 lists extraction ratios and distribution ratios for EPA-TG and DHA-TG in ethanol at different temperatures. Table 2 shows that low temperatures are not advantageous for PUFA-TG extraction, demonstrating that both the solubility of the triglycerides in the ethanol solution and complex formation constants may be facilitated at higher temperatures.

Table 2 Effect of temperature on weight fraction in the extracted oil phase, $X_{\mathrm{i}}$, and distribution ratio, $\mathrm{D}_{\mathrm{i}}$.

\begin{tabular}{ccccc}
\hline Temperature & \multicolumn{2}{c}{ EPA-TG } & \multicolumn{2}{c}{ DHA-TG } \\
& $X_{i}$ & $D_{i}$ & $X_{i}$ & $D_{i}$ \\
\hline $278 \mathrm{~K}$ & 0.20 & 0.32 & 0.11 & 0.51 \\
$288 \mathrm{~K}$ & 0.082 & 1.41 & 0.035 & 2.36 \\
\hline
\end{tabular}

\subsection{Residual copper and counter ion residues}

In Fig. 4, the residual copper in the recovered triglyceride, $W$, is plotted versus the number of washings, $N$. Figure 4 shows that $\log W$ is proportional to $N$, and the following experimental relationship was obtained:

$$
W=W_{0} 10^{-(3 / 4) N}
$$

where $W_{o}$ denotes the amount of copper present before the washing operations. Figure 4 demonstrates that the residual copper was as low as $10 \mathrm{ppm}$ after four washings, and Eq. (7) shows that a $10^{-3 / 4}$ decrease is expected in the residual copper and counter ion, i.e., $\mathrm{NO}_{3}{ }^{-}$, for each washing time. Table 3 lists the compositions of the triglycerides before and after the enrichment operations. Table 3 shows that PUFA-TG can be concentrated from $46 \%$ to $69 \%$ using cuprous ion complex formation extraction and heating recovery.

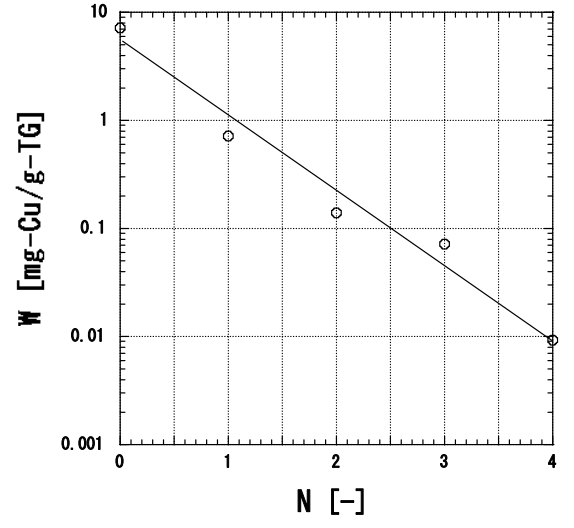

Fig. 4 Relationship between residual copper in the recovered triglyceride, $W$, and the number of washings, $N$.
Table 3 Compositions of the original and PUFA enriched triglycerides.

\begin{tabular}{|c|c|c|}
\hline Component & $\begin{array}{r}\text { original }^{*} \\
\mathrm{wt} \% \\
\end{array}$ & $\begin{array}{r}\text { enriched } \\
\text { wt\% } \\
\end{array}$ \\
\hline $\mathrm{C}_{14: 0}$ & 8.9 & 4.2 \\
\hline$C_{16: 0}$ & 20.2 & 9.9 \\
\hline$C_{18: 0}$ & 14.2 & 6.0 \\
\hline $\mathrm{C}_{20: 5}(\mathrm{EPA})$ & 29.7 & 45.3 \\
\hline $\mathrm{C}_{22: 6}(\mathrm{DHA})$ & 16.8 & 23.8 \\
\hline Others $^{* * *}$ & 10.2 & 10.8 \\
\hline
\end{tabular}

\footnotetext{
*: supplied by Raffinee International Co. Ltd.,

${ }^{* *}:$ after heating recovery

***: including $\mathrm{C}_{12: 0}, \mathrm{C}_{18: 2}$ and $\mathrm{C}_{18: 3}$
} 


\section{Conclusion}

The polyunsaturated fatty acids (PUFA), such as eicosapentaenoic acid and docosahexaenoic acid, contained in fish oil triglycerides were concentrated by cuprous ion complex formation extraction using an extraction solvent consisting of ethanol, cupric nitrate and metallic copper. The equilibration period was as long as six hours reflecting the slow $\mathrm{Cu}(\mathrm{I})$-complex formation. More than $90 \%$ PUFA in the feed triglyceride oil was extracted using a $2 \mathrm{~mol} / \mathrm{dm}^{3} \mathrm{Cu}\left(\mathrm{NO}_{3}\right)_{2}$ ethanol solution at $288 \mathrm{~K}$. The PUFA triglycerides were concentrated from $46 \%$ in the feed stock to $69 \%$ in the product after recovered by heating. The residual copper after four water washings was as low as $10 \mathrm{ppm}$.

\section{Acknowledgement}

The authors are grateful to Raffinee International Co., Ltd., for their financial support to the present investigation.

\section{References}

1) T-J. Lin, S-W. Chen, A-C. Chang, Biochem. Eng. J., 23, 27 (2006).

2) A.P. Haines et al., Thromb. Res., 43, 643 (1986).

3) B.R. Lokesh, J.E. Kinsella, Immunobiology, 175, 406 (1987).

4) D.S. Siscovick, T.E. Raghunathan, I. King, J. Am. Med Assoc., 274, 1363 (1995).

5) J.M. Bourre, O. Dumount, M. Piciotti, G. Pascal. G. Durand, Nutrition, 5, 266 (1989).

6) L.D. Lawson, B.G. Hughes, Biochem. Biophys. Res. Commun., 152, 328 (1988).

7) K. Nakano, R. Maeta, K. Nagahama, S. Kato, Sol. Ext. Res. Dev., Japan, 15, 117 (2008).

8) S. Kato, Y. Mori, S. Tsuchya, K. Nagahama, J. Japan Petroleum Inst., 47, 82 (2004).

9) S. Kato, K. Nakano, H. Noritomi, K. Nagahama, Sol. Ext. Res. Dev., Japan, 3, 117 (1996). 